\title{
EVOLUTION OF DISORDERING IN SiC UPON SINTERING; PHASE ANALYSIS OF SiC BY RIETVELD METHOD WITH APPLICATION OF NEUTRON AND X-RAY DIFFRACTION
}

\author{
B. Palosz \\ High Pressure Research Center, Polish Academy of Sciences \\ Sokołowska 29, 01-142 Warszawa, Poland \\ H. Boysen, J. Schneider and H. Schulz \\ Institut für Kristallographie und Mineralogie der Universität \\ Theresienstrasse 41, 8000 München 2, Germany \\ Dedicated to Professor Dr. Julian Auleytner \\ on the occasion of his 70 th birthday \\ (Received September 18, 1992)
}

\begin{abstract}
Neutron and X-ray diffraction patterns of $\alpha$ and $\beta$ powders as well as of sintered $\mathrm{SiC}$ were analysed by a multiphase Rietveld method. It is shown that structural models combined of large period polytypes can be used to approximate the disordering of these polytype structures. The hexagonality of the samples could be terminated with reproducibility $1-2 \%$ using different combinations of large-period polytypes. It follows that the usual classification in to $\alpha$ and $\beta \mathrm{SiC}$ is an oversimplification. The polytype behaviour of $\mathrm{SiC}$ powders and the role of twinning of cubic layer stackings is discussed.

- Distribution functions of stacking sequences of different length in $\alpha$ and $\beta$ phases are derived.
\end{abstract}

PACS numbers: 61.10.My, 61.12.-q, 61.50.Ks, 81.20.Lb

\section{Introduction}

Silicon carbide is a prominent polytype material and occurs in a number of different structural modifications: periodic and non-periodic (disordered) [1]. It is usually classified as $\alpha$ and $\beta$ SiC. Here $\beta$ represents a cubic phase and $\alpha$ includes various hexagonal and rhombohedral polytypes. For the $\alpha$ phase the contribution of a few basic polytypes: $2 \mathrm{H}, 4 \mathrm{H}, 6 \mathrm{H}$ and $15 \mathrm{R}$ and occasionally disordering of the 
structures was discussed [2-6]. Electron-microscopic studies of individual powder grains show however that there is a variety of multilayer structures with very large periods coexisting with the basic structures $[5,6]$.

Powder diffraction patterns show a small number of dominant reflexions which can all be attributed to the basic structures. It is observed, however, that these reflexions are often broadened with characteristic "diffuse peaks" around some specific reflexions. These diffuse peaks indicate that the structure is disordered and inhomogeneous. Structural changes of polytype structures during sintering are described in the literature, e.g. [4-6].

This work presents an application of the Rietveld method $[7,8]$ to the analysis of disordered polytype structures of $\mathrm{SiC}$ and to the examination of the structural changes occurring in $\alpha$ and $\beta$ powders during sintering. Neutron and X-ray diffraction gives information about the average structure (averaged over all powder grains) and not about local variations of the layer stackings within individual powder grains. (Preliminary results of this work were presented at the International Workshop of the Rietveld Method, Petten, 1989.)

\section{Experimental}

Neutron data were collected on the MAN-I powder diffractometer at the FRM reactor in Garching with a $\mathrm{Cu}(220)$ monochromator, $\lambda=1.075 \AA$ in a step scan mode of interval $0.1^{\circ} 2 \Theta$. Samples were in the form of cylinders $10-15 \mathrm{~mm}$ in diameter, 10-20 $\mathrm{mm}$ high.

X-ray data were collected on a STOE-type diffractometer with a curved $\mathrm{Ge}(111)$ monochromator, and with flat plate of samples coated on foil in transmission geometry and a scintillation counter as a detector. The resulting divergences were $0.5^{\circ}$ horizontally and $1.46^{\circ}$ vertically. Data were collected in a step scan mode at intervals 0.02 and $0.12 \Theta$. The samples are specified in Table I.

TABLE I

Samples specification.

\begin{tabular}{c|c|c}
\hline \hline No. & Type & Source \\
\hline I & $\beta$ powder & STARCK \\
II & $\alpha$ powder & STARCK \\
III & ceramics & \\
& $\alpha$ sintered & STARCK \\
IV & ceramics & \\
& $\beta$ sintered & STARCK \\
V & $\beta$ powder & combustion (SHS) \\
VI & $\beta$ powder & plasma spraying
\end{tabular}




\section{Characteristics of silicon carbide structure}

From single crystal work it is known that there is an almost unlimited number of different structural modifications of SiC: one cubic structure $(3 \mathrm{C})$ and variety of hexagonal $(n \mathrm{H})$ and rhombohedral $(3 n \mathrm{R})$ polytypes and also non-periodic disordered structures [1]. In a first approximation it is assumed that the interlayer spacing (the distance between two subsequent layers $\mathrm{Si}-\mathrm{C}$ ) is preserved (about $2.52 \AA$ ) independent of the stacking, i.e. the layers are always closed packed.

Although there is no clear evidence that some specific layer stackings are more stable than others, it is generally agreed that the layer stackings occurring more frequently than others are the more stable ones. These structures are called basic polytypes: $3 \mathrm{C}-$ cubic, $2 \mathrm{H}, 4 \mathrm{H}$ and $6 \mathrm{H}-$ hexagonal and $15 \mathrm{R}-$ rhombohedral.

According to commonly used $\alpha-\beta$ classification of $\mathrm{SiC}$ materials there should $b e$ the difference between $\alpha$ and $\beta$ related to symmetry between $3 \mathrm{C}$ as cubic i.e. fully isotropic and all other polytype structures which are anisotropic. This classification of $\mathrm{SiC}$ powders into $\beta$ as cubic (3C) and $\alpha$ for others is often misleading. As known from single crystal work and from electron-microscopical observations the pure cubic structure occurs in single crystals only occassionally. It is usually twinned and the same behaviour is expected in powder samples. $3 \mathrm{C}$ occurs in syntactic coalescence with hexagonal and rhombohedral polytypes suggesting that there is a continuous transition between cubic $3 \mathrm{C}$ and other polytype modifications of $\mathrm{SiC}$.

A uniform description of the periodic and disordered structures of $\mathrm{SiC}$ can be given with the use of Zhdanov and hc-notations (Fig. 1) [1]. A single Zhdanov
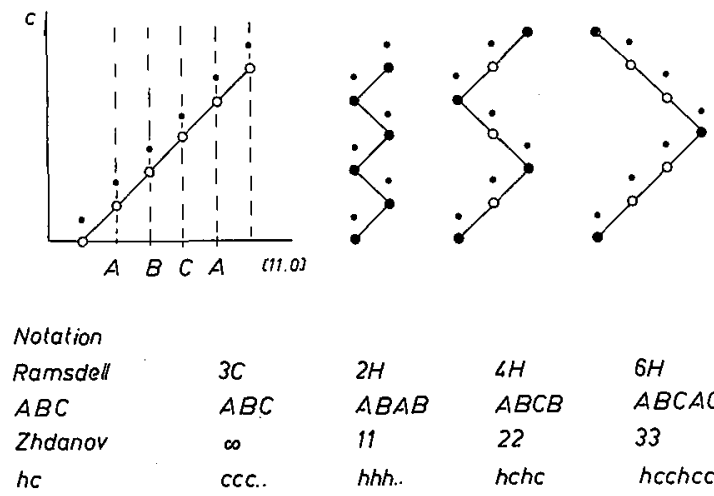

$\begin{array}{ll}4 H & 6 H \\ A B C B & A B C A C B \\ 22 & 33 \\ \text { hchc } & \text { hcchcc }\end{array}$

Fig. 1. Stacking sequences of Si-C layers in the (110) plane of the polytypes $2 \mathrm{H}, 3 \mathrm{C}$, $4 \mathrm{H}, 6 \mathrm{H}$.

number $n_{i}$ represents one domain of the $3 \mathrm{C}$ structure and a change of the stacking from $(-)$ to $(+)$ is equivalent to twinning of the $3 \mathrm{C}$ structure. According to the hc notation a single $\mathrm{SiC}$ layer can have either hexagonal $\mathrm{h}$ or cubic $\mathrm{c}$ surrounding. A single domain of $3 \mathrm{C}$ is a sequence of $c$ layers and one $\mathrm{h}$ layer. A multidomain structure is then combined of blocks of $c$ layers separated by $h$ layers which occur at the interdomain $3 \mathrm{C}(+) / 3 \mathrm{C}(-)$ boundaries. 
From geometrical conditions of close packing there are no restrictions on the relative content of cubic- and hexagonal-like layer stackings. Percent of hexagonal $\mathrm{h}$ layers is called hexagonality and this value for particular structure can be within the range $0-100 \% \mathrm{~h}$.

\section{Data evaluation}

Silicon carbide can be considered as either being combined of pure polytypes (periodic structures) or as a disordered structure which is non-periodic. Note that disordering related to a larger volume does not exclude periodic ordering (i.e. the occurrence of various polytypes) in small parts of the volume, e.g. in single powder grains.

Powder patterns will be very similar for (i) a single very large period polytype, (ii) a combination of several polytypes (a multiphase system) and (iii) randomly disordered structures. When examining polytype structure of $\mathrm{SiC}$ one has to take into account that the lattice constant $a$ is very similar for all polytype structures and the constant $c$ is a multiple of the invariant interlayer distance $c_{0}$ which, in fact, vary very little from polytype to polytype, see e.g. [9-13]. Therefore, the reflexions of individual polytypes occur at similar positions and are often indistinguishable.

In the diffraction patterns of (i) and (ii) there will be very strong overlap of individual (small) reflexions which combine into broad lines looking like single (broadened) reflexions of a small period polytype sitting on a diffuse peak. Similar broadening and diffuse tails of the reflexions will be observed for randomly disordered structures (iii). Note that disordering is in one dimension (the stacking direction) and will give rise to diffuse streaks in single crystals, which in powder show up as characteristic asymmetric diffuse peaks [14].

For structural analysis of the $\mathrm{SiC}$ materials under study we choose the approach (ii) because it seems to be the easiest way to approximate the observed diffraction patterns: (i) would require tests with a very large number of very long period polytypes (and a very large number of atoms in unit cell which exceeds the capacity of standard Rietveld programs), while (iii) would require a special program to account for the characteristic line shapes [14] incorporated into a Rietveld program. On the other hand, (ii) only uses a few polytypes of medium period and can be tackled by a standard multiphase Rietveld program. Only the phase amount (scale factors) have to be refined! We used the version DBWS-9006 of the Rietveld program provided by R.A. Young. In the refinements some structural parameters (temperature factors, atomic positions) were kept fixed and the lattice constants were constrained to simplify multiples of the unit period $c_{0}$. The polytypes used are summarized in Table II, together with corresponding \%h.

Theoretical diffraction patterns calculated for models of twinned cubic structure of two domains of average size of 10-12 Si-C layers, e.g. (8 12), (9 13), (11 12) etc., are nearly identical and do not differ from the pattern calculated for untwinned $3 \mathrm{C}$. In other words, the broadening resulting from twinning of $3 \mathrm{C}$ with the domains of the size 10-12 layers is too small comparing to the instrumental broadening (about $0.4 \mathrm{deg}$ for neutron experiments) to be detected. This 
TABLE II

Polytype structures of $\mathrm{SiC}$ used in the multiphase analysis of $\alpha$ and $\beta$ modifications of SiC.

\begin{tabular}{c|c|c|c}
\hline \hline $\begin{array}{c}\text { Ramsdell } \\
\text { symbol }\end{array}$ & $\begin{array}{c}\text { Zhdanov } \\
\text { sequence }\end{array}$ & $\begin{array}{c}\text { Average twin } \\
\text { stacking }\end{array}$ & $\begin{array}{c}\text { Hexagonality } \\
\% \mathrm{~h}\end{array}$ \\
\hline $2 \mathrm{H}$ & 11 & 1 & 100 \\
$4 \mathrm{H}$ & 22 & 2 & 50 \\
$6 \mathrm{H}$ & 33 & 3 & 33.3 \\
$10 \mathrm{H}$ & 55 & 5 & 20 \\
$11 \mathrm{H}$ & 56 & 5.5 & 18.2 \\
$13 \mathrm{H}$ & 67 & 6.5 & 14.8 \\
$16 \mathrm{H}$ & 88 & 8 & 12.5 \\
$15 \mathrm{R}$ & 23 & 2.5 & 40 \\
$21 \mathrm{R}$ & 34 & 3.5 & 28.6 \\
$24 \mathrm{R}$ & 35 & 4 & 25 \\
$27 \mathrm{R}$ & 45 & 4.5 & 22.2 \\
$3 \mathrm{C}$ & $\infty$ & $\infty$ & 0
\end{tabular}

means that the lowest limit of detection of twinning by this technique and measured as hexagonality is not better than $10 \% \mathrm{~h}$. Clear indication of the presence of reflexion broadening due to stacking disorder is observed from $15 \% \mathrm{~h}$ and very strong changes of the line profiles occur for $20-25 \% \mathrm{~h}$. One should note that the $\alpha-\beta$ classification of polycrystalline $\mathrm{SiC}$ materials based on diffraction techniques is not well substantiated by the content of hexagonal-like layers: there is a wide range of possible values of \%h which are similar for $\alpha$ and for $\beta$, see Discussion.

\section{Results}

The experimental neutron diffraction patterns of $\mathrm{SiC}$ powders are compared in Figs. 2 and 3 to those calculated for multiphase models. Figures $2 \mathrm{a}$ and $3 \mathrm{a}$ present comparison to the patterns calculated for pure polytypes $3 \mathrm{C}$ and $6 \mathrm{H}$, respectively. From this comparison it is clearly seen that the materials under study are not one-phase structures.

With the application of multiphase models the following results were obtained.

\subsection{Sample I ( $\beta$ powder)}

Figure $2 \mathrm{~b}$ presents the neutron diffraction pattern (part of the pattern) which is compared to (a) calculated for pure cubic structure $3 \mathrm{C}$ and to (b) calculated for the multiphase model combined of 6 structures 

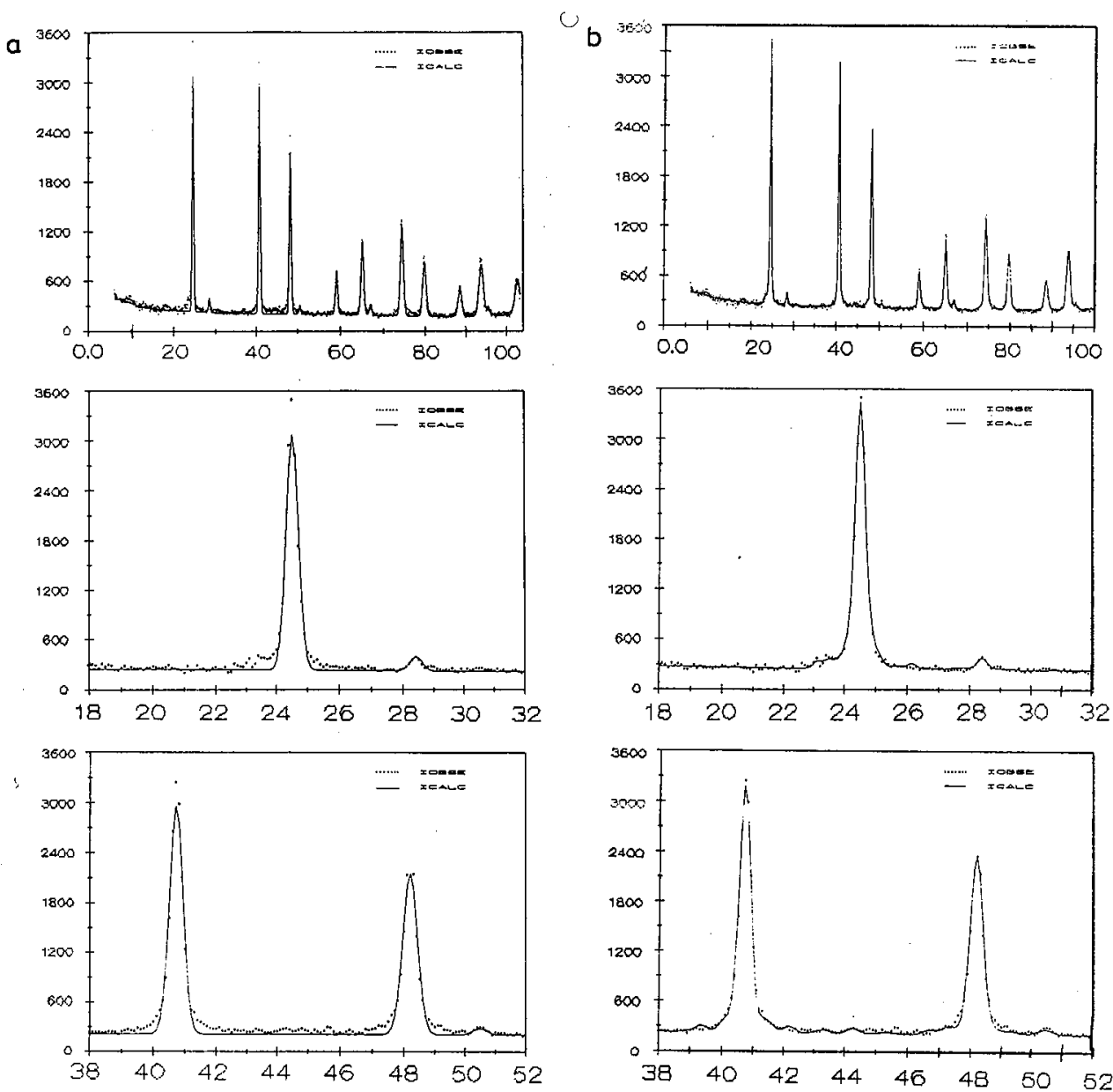

Fig. 2. Neutron diffraction patterns of sample I ( $\beta$ powder): (a) approximation of the experimental pattern by pure cubic $3 \mathrm{C}$ structure (one phase); (b) approximation of the experimental pattern by multiphase model: $3 \mathrm{C}+4 \mathrm{H}+6 \mathrm{H}+10 \mathrm{H}+11 \mathrm{H}+16 \mathrm{H}$.

$$
\begin{aligned}
\text { I. } & 3 \mathrm{C}\left(23.0_{(5)}\right)+4 \mathrm{H}\left(1.0_{(8)}\right)+6 \mathrm{H}\left(4_{(1)}\right) \\
& +10 \mathrm{H}\left(37.0_{(3)}\right)+11 \mathrm{H}\left(25_{(2)}\right)+16 \mathrm{H}\left(11_{(1)}\right)
\end{aligned}
$$

(the values in brackets give the refined content of the polytypes in weight percent together with estimated standard deviations). The total hexagonality of the structure is $15.4 \% \mathrm{~h}$. The values $R_{\mathrm{wp}}$ and $R_{\exp }$ are 9.8 and 6.9 , respectively.

Similar agreements were obtained for models combined of other polytypes, e.g.:

$$
3 \mathrm{C}\left(22.5_{(5)}\right)+10 \mathrm{II}\left(36_{(3)}\right)+11 \mathrm{H}\left(30_{(2)}\right)+13 \mathrm{H}\left(12_{(2)}\right)
$$

where the total hexagonality is $14.4 \% \mathrm{~h}$ and $R_{\mathrm{wp}} / R_{\exp }=10.9 / 6.9$. 


\subsection{Sample II ( $\alpha$ powder)}

Figure 3 presents a comparison of the experimental data to the pattern calculated for pure polytype $6 \mathrm{H} \mathrm{(a)} \mathrm{and} \mathrm{for} \mathrm{multiphase} \mathrm{model} \mathrm{combined} \mathrm{of} 6$ polytypes (b). The model (a) of pure structure $6 \mathrm{H}$ was refined to $R_{\mathrm{wp}} / R_{\exp }=12.2 / 4.3$. Several models were tried to approximate the disordered structure (b):

I. $2 \mathrm{H}\left(0.2_{(3)}\right)+4 \mathrm{H}\left(1.0_{(5)}\right)+6 \mathrm{H}\left(59.7_{(8)}\right)$

$$
+15 \mathrm{R}\left(16_{(2)}\right)+21 \mathrm{R}\left(16_{(2)}\right)+24 \mathrm{R}\left(6_{(1)}\right) .
$$

The total hexagonality of the structure is $33.5 \% \mathrm{~h}, R_{\mathrm{wp}} / R_{\exp }=6.8 / 4.3$.
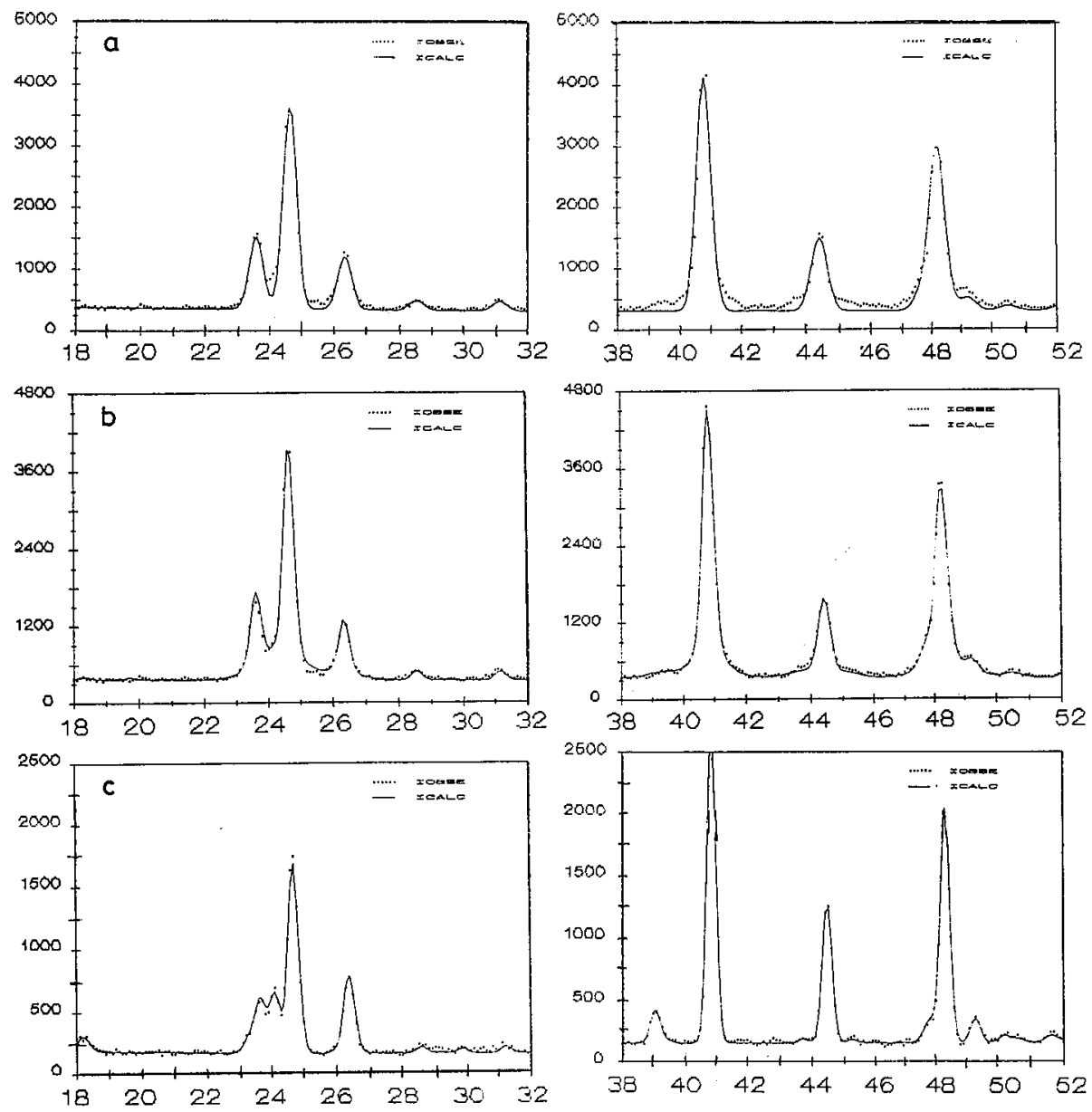

Fig. 3. Neutron diffraction patterns of $\alpha$ phases of SiC: (a) approximation of the experimental pattern of initial $\alpha$ powder, sample II, by pure $6 \mathrm{H}$ structure (one phase); (b) approximation of the powder pattern of sample II by multiphase model: $2 \mathrm{H}+4 \mathrm{H}$ $+6 \mathrm{H}+15 \mathrm{R}+21 \mathrm{R}+24 \mathrm{R}$; (c) approximation of the experimental pattern of sintered $\alpha$, sample III, by the multiphase model: $2 \mathrm{H}+4 \mathrm{H}+6 \mathrm{H}+15 \mathrm{R}+21 \mathrm{R}+24 \mathrm{R}$. 
Similarly good fits of the patterns were also observed for other models, e.g. for the model where the structures $21 \mathrm{R}$ and $24 \mathrm{R}$ were replaced by $10 \mathrm{H}$ and $27 \mathrm{R}$ :

$$
\begin{aligned}
\text { II. } 2 \mathrm{H}\left(0.5_{(8)}\right)+4 \mathrm{H}\left(2.8_{(7)}\right)+6 \mathrm{H}\left(69.2_{(4)}\right) \\
+10 \mathrm{H}\left(2_{(1)}\right)+15 \mathrm{R}\left(13.3_{(8)}\right)+27 \mathrm{R}\left(12_{(1)}\right) .
\end{aligned}
$$

The resulting hexagonality of this model is $33.5 \% \mathrm{~h}$ and $R_{\mathrm{wp}} / R_{\exp }=7.9 / 4.3$.

\subsection{Sample III ( $\alpha$ sintered)}

Figure 3c presents the neutron diffraction pattern of the $\alpha$ powder after sintering. The same multiphase models were calculated for the powder and for the sintered samples but only the model I gave a good fit of the patterns

I. $2 \mathrm{H}(0)+4 \mathrm{H}\left(38_{(1)}\right)+6 \mathrm{H}\left(52.5_{(2)}\right)$

$$
+15 \mathrm{R}\left(5_{(1)}\right)+21 \mathrm{R}\left(25_{(2)}\right)+24 \mathrm{R}\left(1.7_{(4)}\right) \text {. }
$$

The calculated content of $10 \mathrm{H}$ and $27 \mathrm{R}$ for the model II was very small: $0.8_{(8)}$ and $1.2_{(9)}$, respectively. The patterns were fitted very poorly, $R_{\mathrm{wp}} / R_{\exp }=12.5 / 6.2$, which means that this model is inadequate for this particular structure. We note that in this structure having approximately $40 \% \mathrm{~h}$ the average domain contains only 2.5 layers and it is not surprising that the phase analysis shows that there are very few domains 4 and 5 in the structure, cf. Table II $-10 \mathrm{H}$ and $27 \mathrm{R}$.

\subsection{Samples IV, $V$}

Figure 4 presents X-ray patterns of two different samples of $\beta$ powder. The sample IV (Fig. 4a) is similar to sample I but with slightly smaller hexagonality

$$
\begin{aligned}
& 3 \mathrm{C}\left(38.0_{(5)}\right)+4 \mathrm{H}\left(2.1_{(5)}\right)+6 \mathrm{H}\left(1_{(1)}\right) \\
& +10 \mathrm{H}\left(33_{(2)}\right)+11 \mathrm{H}\left(18_{(2)}\right)+16 \mathrm{H}\left(7.4_{(9)}\right) .
\end{aligned}
$$

Total hexagonality is $12.1 \% \mathrm{~h}, R_{\mathrm{wp}} / R_{\exp }=16.6 / 13.6$.

The pattern $4 \mathrm{~b}$ of the sample $\mathrm{V}$ corresponds to another $\beta$ powder which is significantly different from the samples I and IV. Several multipolytype models with different layer stackings were tried, e.g.

I. $2 \mathrm{H}\left(2.9_{(4)}\right)+3 \mathrm{C}\left(5.9_{(8)}\right)+4 \mathrm{H}\left(2_{(3)}\right)+6 \mathrm{H}\left(11_{(2)}\right)$

$$
+10 \mathrm{H}\left(28_{(9)}\right)+11 \mathrm{H}\left(15_{(2)}\right)+21 \mathrm{R}\left(23_{(3)}\right)+24 \mathrm{R}\left(14_{(2)}\right)
$$

with total hexagonality $26.1 \% \mathrm{~h}, R_{\mathrm{wp}} / R_{\exp }=20.5 / 16.4$,

$$
\text { II. } \begin{aligned}
& 2 \mathrm{H}\left(2.7_{(3)}\right)+3 \mathrm{C}\left(6.1_{(6)}\right)+4 \mathrm{H}\left(3_{(1)}\right)+6 \mathrm{H}\left(6_{(2)}\right) \\
+ & 10 \mathrm{H}\left(58_{(5)}\right)+11 \mathrm{H}\left(7_{(3)}\right)+15 \mathrm{R}\left(17_{(2)}\right)
\end{aligned}
$$

with total hexagonality $28.3 \% \mathrm{~h}, R_{\mathrm{wp}} / R_{\exp }=17 / 13.3$.

Note: Hexagonality $26-28 \% \mathrm{~h}$ means that the average domain in this structure is about 3.7 layers. Despite the large hexagonality, one observes only the intensity maxima which can be attributed to the cubic structure 3C. A poor fit 

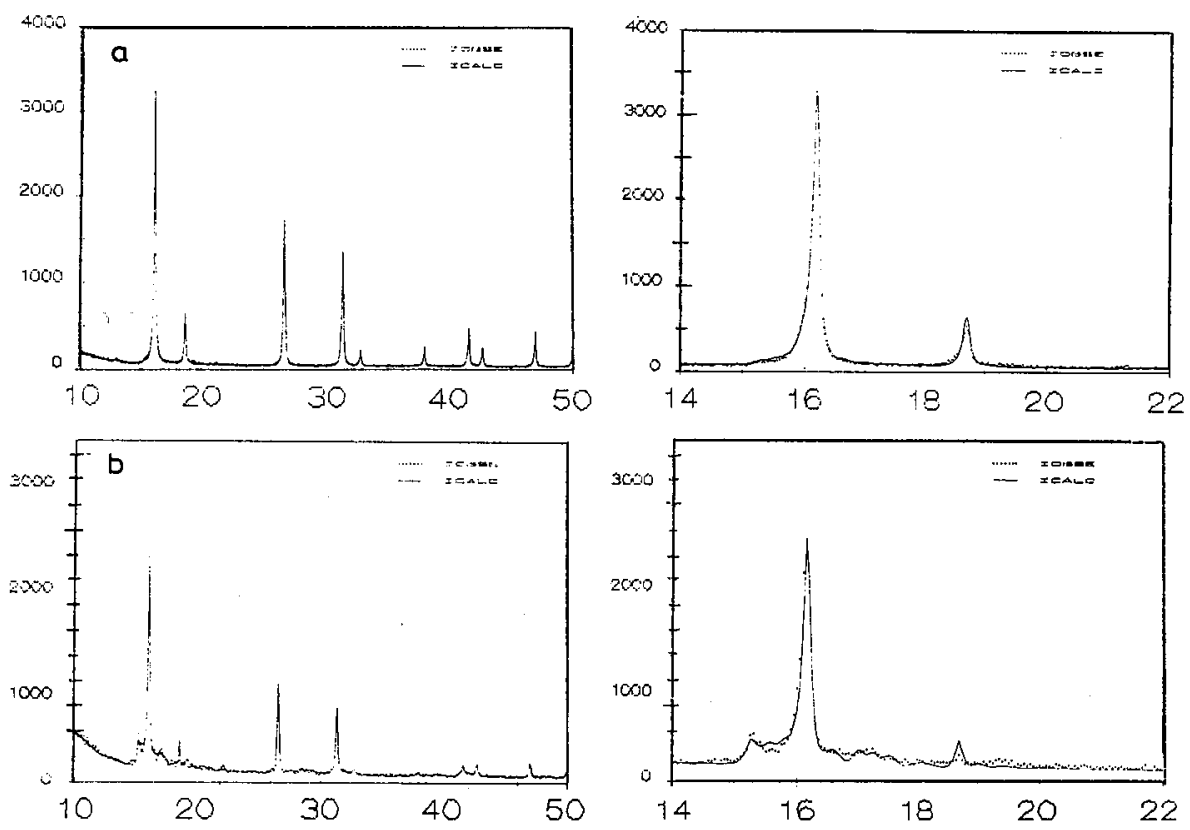

Fig. 4. X-ray diffraction pattern of $\beta$ powders: (a) sample $V$, (b) sample VI by multiphase model: $2 \mathrm{H}+3 \mathrm{C}+4 \mathrm{H}+6 \mathrm{H}+10 \mathrm{H}+16 \mathrm{H}$.

of the $3 \mathrm{C}$ reflexions suggests that either the sample is textured or the shape of the grains is strongly anisotropic. This sample shows very clearly how much the classification of the $\mathrm{SiC}$ powders into $\alpha$ and $\beta$ can be misleading: the sample VI classified as $\beta$ with about $27 \% \mathrm{~h}$ is more $\alpha$-like than cubic structure. At the same time the pattern of this structure exhibits no similarities to typical $\alpha$ patterns of Fig. 3.

\section{Discussion}

Figures 5 and 6 present distribution functions of the sizes of the domains of the $3 \mathrm{C}$ twins in $\alpha$ and $\beta$ powders as derived from the phase analysis (relative content of different domains given as Zhdanov numbers). The multiphase model allows the calculation of only a few discrete points of the function for particular values of \%h. In Fig. 5 the values derived from different models for the same powder sample are plotted together. These points fit well into one distribution function which proves that the derivation of the distribution function is independent from the chosen polytype composition.

Accepting the model of the polytype structures of the $\mathrm{SiC} \beta$ powders as resulting from the twinning of $3 \mathrm{C}$, one accepts that there are no forces which could lead to periodic ordering. Under this assumption the twinning should be random. If this is true, the frequency of occurrence of domains of different sizes should be described by a statistical distribution function. In case of random disordering it 


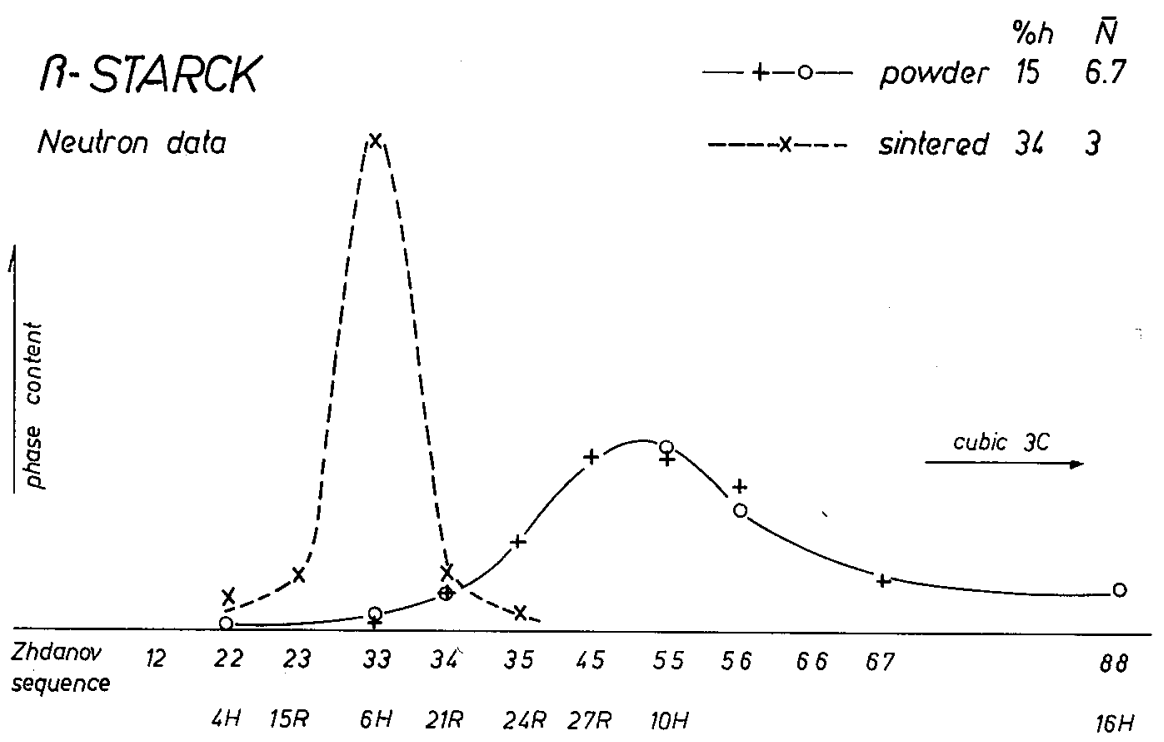

Fig. 5. Distribution of stacking sequences in the structure of the initial $\beta$ powder (sample I) and in this powder after sintering (sample IV). Note: Two sets of the points calculated for two different models are plotted: $+++: 3 \mathrm{C}+6 \mathrm{H}+11 \mathrm{H}+13 \mathrm{H}+21 \mathrm{R}+24 \mathrm{R}$, $000: 3 \mathrm{C}+4 \mathrm{H}+6 \mathrm{H}+10 \mathrm{H}+11 \mathrm{H}+21 \mathrm{R}$.

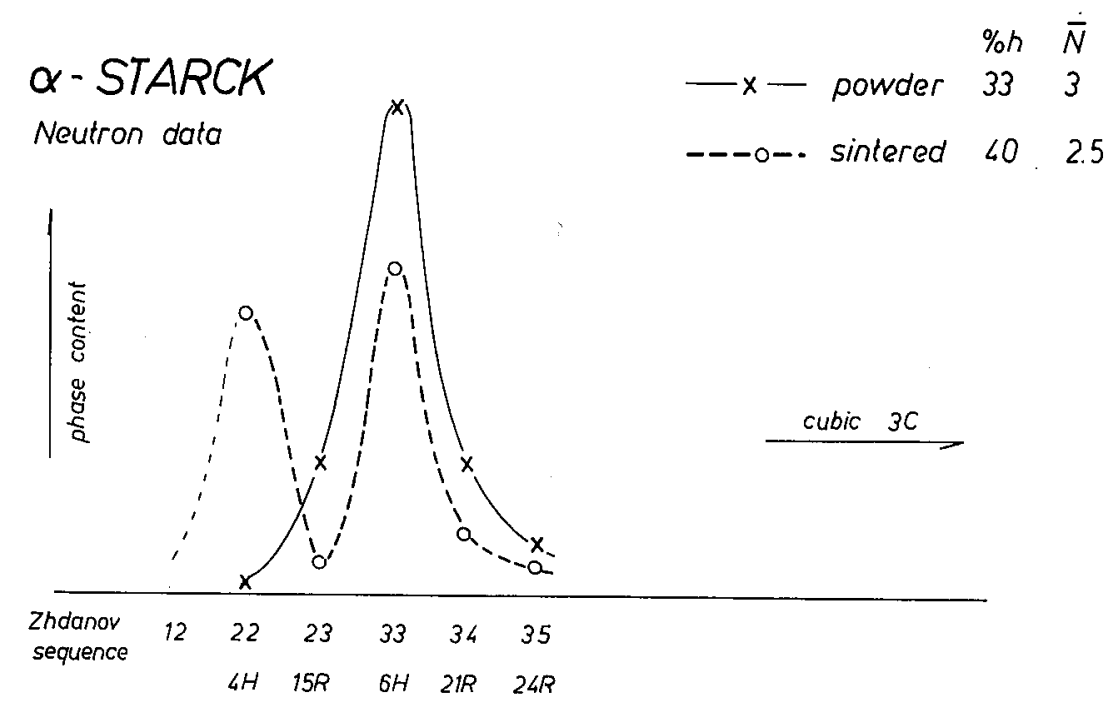

Fig. 6. Distribution of the stacking sequences in the structure of initial $\alpha$ powder (sample II) and this powder after sintering (sample III). 
should be a Gaussian-type normal distribution function. The plots presented in Figs. 5 and 6 for $\alpha$ and $\beta$ powders respectively are indeed similar to normal distribution function. However, the experimental function of $\beta$ powder is not symmetric as one could expect. The parts of the curves corresponding to the larger domains are elongated which can be explained as due to the approximation of the twinned $3 \mathrm{C}$ structure with the domains larger than 8 by pure $3 \mathrm{C}$.

Figure 5 compares the distribution functions of $\beta$ powder before and after sintering. The hexagonality of the structure increases upon sintering from about 15 to $33.5 \% \mathrm{~h}$ and the distribution functions of the initial and of the resulting structures are normal-type functions. The structural changes are then the transition of the initially randomly twinned structure $3 \mathrm{C}$ into another randomly disordered structure but with much smaller domains.

Figure 6 compares the distribution functions of $\alpha$ powder before and after sintering. The distribution function of the $\alpha$ powder is a symmetrical normal function as expected for the randomly disordered structure. The distribution function of the sintered material is split into two maxima corresponding to $4 \mathrm{H}$ and $6 \mathrm{H}$ stackings (22) and (33). This split can be explained by the following. In the initial $\alpha$ powder with $33.5 \% \mathrm{~h}$ the average twin size is only 3 layers and the stacking se.quence is given mainly by the domains $2,3,4$ and 5 . During sintering the individual domains divide into smaller ones. The development of the twinning, however, is possible only in the domains larger than 3: the domains 2 do not undergo further division into smaller domains because this domain ( 2 corresponds to he stacking) is already the smallest single twin of $3 \mathrm{C}$. The domains 3 also do not undergo further twinning because the division of this domain would require the creation of two domains: 1 and 2; of those the singular domain 1 does not occur, see above. The twinning occurs then only in the domains 4,5 and larger and can develop only until the smallest domains are 2 and 3. The resulting structure of sintered $\alpha$ or $\beta$ is very much enriched in the stackings 2 and 3 compared to the initial powders, which agrees very well with the distribution function of Fig. 6 .

\section{Final remarks and conclusions}

In the present work we concentrated on the identification of the layer stackings in the $\alpha$ and $\beta$ modifications of SiC powders and on the determination of the phase composition of the powders and of the sintered SiC. In general, a model of randomly disordered structure should include possibly large spectrum of the sizes of the domains which can have from 1 to an infinite number of the layers in a single domain. The calculations which we performed were limited to relatively simple models where the largest domain in the polytype cell was of 8 layers $16 \mathrm{H}$, Table II. Limitation of the maximum size of the domains in our models to 8 and approximation of other twinned structures by untwinned $3 \mathrm{C}$ may lead to a small underestimation of $\% \mathrm{~h}$, e.g. up to $2.5 \% \mathrm{~h}$ in $\beta$ powder (sample I) and only $0.5 \% \mathrm{~h}$ in sintered materials, e.g. sample II.

Performing the phase analysis we assumed that the lattice sites are fully occupied whatever the layer stacking is. There are indications, however, that in the structure of different polytypes there are certain deviations from stoichiometry 
of $\mathrm{SiC}$ and, moreover, the polytype structure is strongly influenced by impurities, e.g. $[10,11]$.

The thermal parameters taken for the Rietveld analysis were fixed to $B_{\mathrm{Si}}=0.1$ and $B_{\mathrm{C}}=0.2$ and were not refined. The phase composition derived from the refinements with isotropic as above and with anisotropic parameters taken from single crystal data [13] were almost the same.

The results given here present a rather easy and quick way of characterizing polycrystalline (powdered or sintered) SiC samples. This work is now continued into direction of performance of quantitative phase analysis which will include the crystalline polytype phases and also non-crystalline phases present in polycrystalline $\mathrm{SiC}$, in particular nanocrystals. For preliminary results see $[15,16]$.

\section{References}

[1] A.R. Verma, P. Krishna, Polymorphism and Polytypism in Crystals, Wiley, New York 1966.

[2] J. Ruska, L.J. Gauckler, J. Lorenz, H.U. Rexer, J. Mater. Sci. 14, 2013 (1979).

[3] M. Lancin, J. Mater. Sci. 19, 4077 (1984).

[4] P. Greil, D. Stutz, Lecture Meeting on "Advanced Ceramics", Tokyo Institute of Technology, Yokohama, Japan 1983.

[5] L.U. Ogbuji, T.E. Mitchell, A.H. Heuer, J. Am. Ceram. Soc. 64, 91 (1981).

[6] R.M. Williams, B.N. Juterbock, S.S. Shinozaki, C.R. Peters, T.J. Whalen, Am. Ceram. Soc. Bull. 64, 1385 (1985).

[7] H.M. Rietveld, Acta Crystallogr. 22, 151 (1967).

[8] H.M. Rietveld, J. Appl. Crystallogr. 2, 65 (1969).

[9] Yu.A. Vodakov, G.A. Lomakina, E.N. Mokhov, Sov. Phys. Solid State 24, 780 (1982).

[10] N.D. Sorokin, Yu.M. Tairov, V.F. Tsvetkov, M.A. Chernov, Sov. Phys.-Crystallogr. 28, 539 (1983).

[11] K.L. More, J. Ryu, C.H. Carter Jr., J. Bentley, R.F. Davis, Cryst. Latt. Def. Amorph. Mat. 12, 243 (1985).

[12] J. Guth, W.T. Petuskey, J. Phys. Chem. Solids 48, 541 (1987).

[13] K. Seebauer, W. Steurer, B. Pałosz, in: Tagung der Arbeitsgemeinshaft für Kristallographie, München 1991, R. Oldenbourg Verlag, München 1991, p. 257.

[14] H. Boysen, J. Appl. Crystallogr. 18, 320 (1985).

[15] B. Bondars, S. Gierlotka, B. Pałosz, in: EPDIC-2, Enschede 1992, in press.

[16] B. Pałosz, S. Gierlotka, F. Guignard, L. Stobierski, R. Pampuch, in: Jahrestagung der DGK, Mainz 1992, R. Oldenbourg Verlag, München 1992, p. 179. 